\title{
SYSGRAN: UM SISTEMA DE CÓDIGO ABERTO PARAANÁLISES GRANULOMÉTRICAS DO SEDIMENTO
}

\author{
MAURÍCIO GARCIA DE CAMARGO
}

\begin{abstract}
Resumo SysGran é um software para a análise granulométrica de sedimentos, contando com uma rica saída gráfica. O software é agora é disponibilizado sob a licença GNU, o que significa que, além de completamente gratuito, pode ser livremente modificado e aperfeiçoado. O software foi desenhado para maximizar a velocidade das análises, sendo capaz de analisar centenas de amostras em poucos segundos e de gerar rapidamente uma vasta gama de gráficos. Os resultados das análises compreendem todas as estatísticas do sedimento por diversos métodos gráficos como Folk \& Ward ou a medida dos momentos (média, mediana, seleção, assimetria, curtose), as frações granulométricas (percentual de cascalho, areia, silte e argila), classificação verbal, os principais percentís e os dados para plotagem da curva acumulada em outros programas gráficos.
\end{abstract}

Palavras-chave: software, código livre, sedimento, granulometria.

\begin{abstract}
SYSGRAN: AN OPEN SOURCE SYSTEM FOR GRAIN SIZE ANALYSIS OF SEDIMENT. SysGran is an easy-to-use specific software for granulometric analysis of sediments, which includes a rich graphical output. The software is now available under GNU license, meaning that it is free for any use and can be freely modified and improved. The software has been designed to maximize speed of analysis and is able to analyze hundreds of samples in a few seconds, together with many types of graphics. The results of statistical analysis of the sediment includes Folk \& Ward graphical methods (among others) as well as methods of moments (mean, median, sorting, skewness, curtosis), grain size fractions (gravel, sand, silt and clay percentages), verbal classification, main percentile units and data for cumulative frequency curve plot using other graphical softwares.
\end{abstract}

Keywords: software, open source, sediments, grain size.

INTRODUÇÃO A principal propriedade de um sedimento é o tamanho das suas partículas, que afeta as condições deposicionais e de transporte, podendo ser utilizado como uma ferramenta básica de caracterização de solos com o propósito de interpretar os ambientes de sedimentação (Folk \& Ward, 1957; Friedman, 1979; Sugita \& Maruno, 2001). Mais especificamente, a análise do tamanho do grão é aplicada para correlacionar amostras provenientes de diferentes unidades estratigráficas, determinar o agente de transporte e deposição (vento, rio, corrente de turbidez, etc) e determinar processos de deposição final para caracterizar o ambiente de sedimentação (Carver, 1971). Um simples mapa da percentagem de areia no sedimento poderia indicar a existência de algum gradiente relacionado a um possível padrão de distribuição do tipo de sedimento.

Existem diversas técnicas para determinação do tamanho do grão, como medição direta, peneiramento seca e úmida, sedimentação, granulometria a laser, raio-X e Coulter Counter (McManus, 1988), cada uma mais apropriada para determinadas faixas de tamanho de grão. Todos os métodos envolvem a divisão das amostras de sedimento em um número menor de frações de tamanho, possibilitando a construção de uma distribuição de freqüência de tamanho baseada no peso ou volume percentual de cada categoria (Pye \& Blott, 2004). O número e os limites destas categorias variam de acordo com o método da análise: peneiração resulta em poucas categorias (peneiras) enquanto a granulometria a laser possibilita a aplicação de centenas de categorias.

Um tratamento matemático dos dados de distribuição de tamanho do grão se torna então necessário para a caracterização das amostras. Existem basicamente três tipos de manipulações matemáticas possíveis para os dados: a) interpretação visual da distribuição, através de histogramas, curvas de distribuição de frequiências e outras interpolações; b) cálculo dos parâmetros estatísticos (média, desvio padrão, assimetria e curtose) a partir dos dados originais de percentagens (método dos momentos); e c) cálculo dos parâmetros estatísticos a partir de interceptos tirados visualmente a partir dos gráficos ou por métodos de interpolação-regressão (métodos gráficos). Através do SysGran, a completa caracterização das amostras é possível através de todos estes métodos (e alguns outros), de uma maneira muito rápida e precisa.

\section{CARACTERÍSTICAS DO SOFTWARE}

- Nome: SysGran para Windows;

- Desenvolvimento iniciado por: Maurício Garcia de Camargo;

- Ano de início do desenvolvimento: 1999;

- Licença atual (a partir de 2006): GNU;

- Disponibilidade: www.cem.ufpr.br/sysgran;

- Custo: Nenhum;

- Limite de amostras: 16.384

- Limite de classes: 256 (permite análise de granulometria a laser)

- Arquivo de instalação binária: sysgran.zip $(4,3 \mathrm{Mb})$;

- Arquivo do código fonte: SysGran3_Source.zip $(107 \mathrm{~Kb})$;

- Hardware mínimo: Intel x86 com no mínimo 128 MB de memória RAM;

- Plataformas testadas: Windows 98, ME, 2000, XP.

- Linguagem de programação: Delphi 5.

\section{ARQUITETURA DO SYSGRAN}

As planilhas As planilhas do SysGran são bastante parecidas com aquelas do Excel ( ${ }^{*}$.xls). De fato, as planilhas são salvas no formato do Excel. O SysGran é capaz de ler e gravar diretamente planilhas do Excel versões 4.0 e 5.0. Infelizmente, ainda não há suporte para as planilhas mais modernas do Excel, a partir 
Tabela 1. Arranjo da planilha de entrada de dados no SysGran (exemplo), com as classes de tamanho de grão ( $\phi)$ nas colunas e as amostras identificadas individualmente nas linhas.

\begin{tabular}{l|cccccc}
\hline & $-1,0$ & $-0,5$ & 0 & 0,5 & 1,0 & 1,5 \\
\hline Amostra1 & 0 & 0 & 0,76 & 0,90 & 1,65 & 15,87 \\
Amostra2 & 0 & 0,84 & 1,50 & 2,80 & 6,76 & 22,54 \\
Amostra3 & 0 & 1,23 & 2,10 & 5,32 & 12,87 & 18,65 \\
Amostra4 & 0 & 0,23 & 4,76 & 8,65 & 16,43 & 12,45 \\
\hline
\end{tabular}

da versão 7.0. Quem possui uma destas versões deve salvar as planilhas como Excel versão 4.0 para evitar possíveis problemas de compatibilidade.

As planilhas de entrada de dados devem ser arranjadas de acordo com a tabela 1 .

O SysGran reconhece o fim da seqüência de amostras e classes de tamanho a partir da primeira célula em branco. Portanto, nenhuma linha ou coluna em branco dentro bloco a ser analisado deve permanecer vazia.

Na primeira linha entra-se com as classes de $\phi$ (phi) e na primeira coluna com a identificação única das amostras. O SysGran dá ênfase à velocidade de processamento de muitas amostras e à facilidade de uso, e para isso não se propõe a funcionar como uma unidade armazenadora de dados. A identificação única da amostra (até 12 caracteres) deve ser estabelecida a priori, e esta será a única referência da amostra para o programa.

O SysGran permite o uso de classes padrão, que podem ser digitadas e gravadas para posterior utilização em outras planiThas (menu Planilhas>Adicionar/Capturar classes padrão à primeira linha).

Operações padrão com planilhas estão disponíveis a partir do menu principal.

As análises As análises estatísticas do sedimento foram baseadas em Suguio (1973) e Tanner (1995). Cinco métodos gráficos de análise estão disponíveis: Folk \& Ward, McCammon (a), McCammon (b), Trask, Otto \& Inman, e um não gráfico (algébrico), as Medidas dos Momentos, sendo padrão o primeiro (figura 1).

A primeira decisão a ser feita na opção de análise é entre dados brutos e estatística do sedimento. Dados brutos da curva acumulada resultam em uma planilha com valores para cada classe de phi dos pesos proporcionais, das percentagens e das percentagens acumuladas de cada amostra, que podem ser exportados para outros programas e utilizados como fonte de dados para gráficos de natureza variada. A análise estatística do sedimento (figura 3 ) pode conter apenas valores da média, seleção, mediana, curtose, assimetria, \% de areia, \% de argila e \% de silte, ou incluir opcionalmente classificações verbais e percentis (de $03 \%$ até $97 \%$ ).

Todos os métodos e procedimentos analíticos são detalhados na tabela 2, que foi baseada em Suguio (1973). Nestas informações técnicas, incluíram-se as "taxas de eficiência" de cada método para a média e seleção, que são a razão de acerto em relação à média e desvio padrão reais.

Os valores da média do tamanho do grão são usados para categorizar os sedimentos através de métodos propostos por diversos autores (tabela 3). A escala mais antiga é a de Wentworth (1922) e todas as demais são adaptações desta, . Adota-se aqui a escala de Wentworth stritu sensu com a modificação semântica do nome ultra-argila para argila. Os limites são dados em valores de phi $(\phi)$, obtidos pela fórmula abaixo a partir de valores em milímetros:

$$
(p h i)(\phi)(f i)=-\log _{2} d(m m)
$$

Os gráficos O SysGran possui uma capacidade gráfica bastante razoável (figura 2), com gráficos univariados (histogramas) que podem ser analisados seqüencialmente (para análise modal), bivariados entre pares de parâmetros estatísticos, e multivariados, como o de freqüência acumulada ou de probabilidades, que aceitam no máximo 10 amostras plotadas conjuntamente, ou ainda os diagramas triangulares de Shepard e Pejrup (figura 4). $O$ diagrama triangular de Pejrup constitui uma maneira de se avaliar as condições hidrodinâmicas dos locais onde as amostras foram tomadas (Pejrup, 1988).

O limite de amostras para os diagramas triangulares é o mesmo do número máximo de amostras (16.384), mas muitas amostras plotadas simultaneamente podem gerar um gráfico confuso. De fato, um diagrama com centenas de amostras plotadas não apresenta nenhuma utilidade. Os algoritmos para se construir os diagramas são pesados e para muitas amostras a visualização pode levar um tempo considerável. Os plots nos diagramas são mostrados em azul, de acordo com uma simbologia especial. Triângulos representam a quantidade de grânulos na amostra, que é a quarta variável. Quando esta quantidade é muito pequena $(<3 \%)$, o triângulo também é pequeno e não aparece, sendo substituído por um círculo.

Todos os gráficos podem ser salvo em formato BMP, WMF e EMF, impresso ou copiados para o Clipboard e transferidos para outros programas.

DISCUSSÃO O SysGran foi projeto para maximizar a velocidade de análise das amostras. Após a entrada dos dados, o programa é capaz de analisar centenas de amostras em poucos segundos. Antigos programas para MS-DOS (Granulo e Angra) não dispunham de entrada de dados massiva nem possuíam a capacidade de análise seqüencial, o que tornava muito moroso o processo com muitas amostras. Programas baseados em VBA (Microsoft Visual Basic for Applications) integrados ao Excel (Gradistat e Granplott) analisam sequencialmente as amostras, mas são reconhecidamente lentos; o programa Gradistat, segundo os autores (Blott \& Pye, 2001), pode calcular aproximadamente 50 amostras por hora. Mesmo com uma saída de dados que inclui alguns gráficos (e que portanto atrasam o resultado), o SysGran é algumas centenas de vezes mais rápido. Quanto à velocidade de análise, o SysGran se iguala a produtos comerciais, como ANASED e WinSieve.

Mesmo com todas as facilidades introduzidas pelo SysGran, cabe ao usuário interpretar os resultados de maneira apropriada para a hipótese do trabalho. Para isso, algumas precauções devem ser tomadas. O modelo estatístico no qual todos os parâmetros são estimados é baseado na distribuição normal e, portanto, amostras com grandes desvios desta distribuição não podem ser corretamente interpretadas. O exemplo típico são amostras com mais de uma moda (multimodais), que não permitem a correta 
Tabela 2 - Fórmulas estatísticas disponiveis no SysGran utilizadas para calcular a) graficamente e b) numericamente (medidas dos momentos) os parâmetros estatísticos dos sedimentos, com suas respectivas classificações verbais.

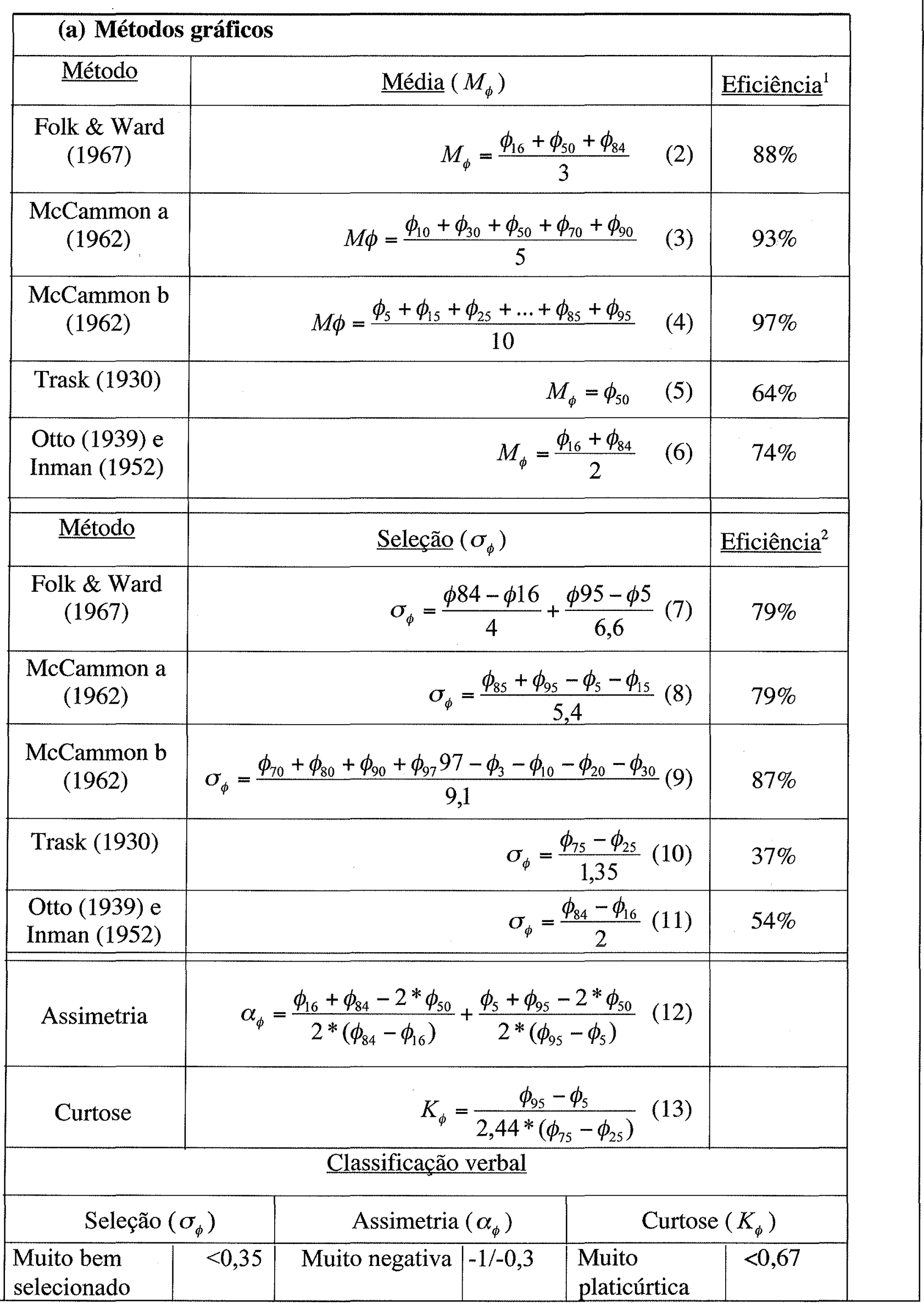


Tabela 2 - continuação

\begin{tabular}{|c|c|c|c|c|c|}
\hline Bem selecionado & $0,35 / 0,5$ & Negativa & $-0,3 /-0,1$ & Platicúrtica & $0,67 / 0,9$ \\
\hline $\begin{array}{l}\text { Moderadamente } \\
\text { selecionado }\end{array}$ & $0,5 / 1,0$ & $\begin{array}{l}\text { Aproximada- } \\
\text { mente } \\
\text { simétrica }\end{array}$ & $-0,1 / 0,1$ & Mesocúrtica & $0,9 / 1,11$ \\
\hline $\begin{array}{l}\text { Pobremente } \\
\text { selecionado }\end{array}$ & $1,0 / 2,0$ & Positiva & $0,1 / 0,3$ & Leptocúrtica & $1,11 / 1,5$ \\
\hline $\begin{array}{l}\text { Muito } \\
\text { pobremente } \\
\text { selecionado }\end{array}$ & $2,0 / 4,0$ & Muito positiva & $0,3 / 1,0$ & $\begin{array}{l}\text { Muito } \\
\text { Leptocúrtica }\end{array}$ & $1,5 / 3$ \\
\hline $\begin{array}{l}\text { Extremamente } \\
\text { mal selecionado }\end{array}$ & $>4$ & & & $\begin{array}{l}\text { Extremamente } \\
\text { leptocúrtica }\end{array}$ & $>3$ \\
\hline
\end{tabular}

(b) Método dos momentos ${ }^{3}$

\begin{tabular}{r|r|r|l}
\hline \multicolumn{1}{l|}{$\begin{array}{l}\text { Média } \\
\bar{x}_{\phi}=\frac{\sum f m_{\phi}}{100}\end{array}$} & $\begin{array}{l}\text { Seleção } \\
\sigma_{\phi}=\sqrt{\frac{\sum f\left(m_{\phi}-\bar{x}_{\phi}\right)^{2}}{100}}\end{array}$ & $\begin{array}{l}\text { Assimetria } \\
s k_{\phi}=\frac{\sum f\left(m_{\phi}-\bar{x}_{\phi}\right)^{3}}{100 \sigma_{\phi}^{3}}\end{array}$ & \multicolumn{1}{l}{ Curtose } \\
$K_{\phi}=\frac{\sum f\left(m_{\phi}-\bar{x}_{\phi}\right)^{4}}{100 \sigma_{\phi}^{4}}$ \\
$(14)$ & & $(16)$ &
\end{tabular}

Onde:

$f=$ percentagem do peso de cada freqüência para cada tamanho de grão;

$\boldsymbol{m}=$ mediana de cada graduação de tamanho de grão em phi $(\phi)$

\begin{tabular}{|c|c|c|c|c|c|}
\hline \multicolumn{6}{|c|}{ Classificação verbal } \\
\hline \multicolumn{2}{|c|}{ Seleção $\left(\sigma_{\phi}\right)$} & \multicolumn{2}{|c|}{ Assimetria $\left(\alpha_{\phi}\right)$} & \multicolumn{2}{|c|}{ Curtose $\left(K_{\phi}\right)$} \\
\hline $\begin{array}{l}\text { Muito bem } \\
\text { selecionado }\end{array}$ & $<0,35$ & Muito negativa & $<-1,3$ & \begin{tabular}{|l|}
$\begin{array}{l}\text { Muito } \\
\text { platicúrtica }\end{array}$ \\
\end{tabular} & $<1,70$ \\
\hline Bem selecionado & $0,35 / 0,5$ & Negativa & $\begin{array}{l}-1,3 /- \\
0,43\end{array}$ & Platicúrtica & $1,7 / 2,55$ \\
\hline $\begin{array}{l}\text { Moderadamente } \\
\text { selecionado }\end{array}$ & $0,5 / 1,0$ & $\begin{array}{l}\text { Aproximada- } \\
\text { mente } \\
\text { simétrica }\end{array}$ & $\begin{array}{l}- \\
0,43 / 0,43\end{array}$ & Mesocúrtica & $2,55 / 3,7$ \\
\hline $\begin{array}{l}\text { Pobremente } \\
\text { selecionado }\end{array}$ & $1,0 / 2,0$ & Positiva & $0,43 / 1,3$ & Leptocúrtica & $3,7 / 7,4$ \\
\hline $\begin{array}{l}\text { Muito } \\
\text { pobremente } \\
\text { selecionado }\end{array}$ & $2,0 / 4,0$ & Muito positiva & $>1,3$ & $\begin{array}{l}\text { Muito } \\
\text { Leptocúrtica }\end{array}$ & $7,4 / 15$ \\
\hline $\begin{array}{l}\text { Extremamente } \\
\text { mal selecionado }\end{array}$ & $>4$ & & & $\begin{array}{l}\text { Extremamente } \\
\text { leptocúrtica }\end{array}$ & $>15$ \\
\hline
\end{tabular}

1 Eficiência testada por MacCammon (1962)

2 Eficiência testada por Folk \& Ward (1966)

3 Extraído de Tanner (1995) 
Tabela 3. Comparação de diferentes escalas de tamanho adotadas por diferentes autores. O programa SysGran utiliza uma escala ligeiramente adaptada (veja o texto) de Wentworth (1922), obtida de Suguio (1973). Esta escala é uma das mais adotadas por laboratórios brasileiros.

\begin{tabular}{|c|c|c|c|c|}
\hline \multicolumn{2}{|c|}{ Tamanho do Grão } & \multicolumn{3}{|c|}{ Classificação verbal } \\
\hline$f i(p h i)(\phi)=-\log _{2} d(m m)$ & $\mathrm{mm} / \mu \mathrm{m}$ & $\begin{array}{l}\text { Wentworth } \\
(1922) \\
\end{array}$ & $\begin{array}{c}\text { Friedman \& } \\
\text { Sanders (1978) }\end{array}$ & $\begin{array}{c}\text { Blott \& Pye } \\
(2001)\end{array}$ \\
\hline$<-11$ & $\begin{array}{c}>2048 \\
\mathrm{~mm}\end{array}$ & \multirow{4}{*}{ Matacão } & $\begin{array}{c}\text { Matacão muito } \\
\text { grande }\end{array}$ & \\
\hline$-10 \rightarrow-11$ & $\begin{array}{c}1024 \rightarrow 20 \\
48\end{array}$ & & Matacão grande & $\begin{array}{c}\text { Calhau muito } \\
\text { grande }\end{array}$ \\
\hline$-9 \rightarrow-10$ & $\begin{array}{c}512 \rightarrow 102 \\
4\end{array}$ & & Matacão médio & Calhau grande \\
\hline$-8 \rightarrow-9$ & $256 \rightarrow 512$ & & $\begin{array}{l}\text { Matacão } \\
\text { pequeno }\end{array}$ & Calhau médio \\
\hline$-7 \rightarrow-8$ & $128 \rightarrow 256$ & \multirow[b]{2}{*}{ Bloco } & Calhau grande & Calhau pequeno \\
\hline$-6 \rightarrow-7$ & $64 \rightarrow 128$ & & Calhau pequeno & $\begin{array}{c}\text { Calhau muito } \\
\text { pequeno }\end{array}$ \\
\hline$-5 \rightarrow-6$ & $32 \rightarrow 64$ & \multirow{4}{*}{ Seixo } & $\begin{array}{l}\text { Seixo muito } \\
\text { grosso }\end{array}$ & $\begin{array}{c}\text { Granulo muito } \\
\text { grosso }\end{array}$ \\
\hline$-4 \rightarrow-5$ & $16 \rightarrow 32$ & & Seixo grosso & Granulo grosso \\
\hline$-3 \rightarrow-4$ & $8 \rightarrow 16$ & & Seixo médio & Granulo médio \\
\hline$-2 \rightarrow-3$ & $4 \rightarrow 8$ & & Seixo fino & Granulo fino \\
\hline$-1 \rightarrow-2$ & $2 \rightarrow 4$ & Granulo & $\begin{array}{l}\text { Seixo muito } \\
\text { fino }\end{array}$ & $\begin{array}{l}\text { Granulo muito } \\
\text { fino }\end{array}$ \\
\hline $0 \rightarrow-1$ & $1 \rightarrow 2$ & $\begin{array}{c}\text { Areia muito } \\
\text { grossa }\end{array}$ & $\begin{array}{c}\text { Areia muito } \\
\text { grossa }\end{array}$ & $\begin{array}{c}\text { Areia muito } \\
\text { grossa }\end{array}$ \\
\hline $1 \rightarrow 0$ & $\begin{array}{c}500 \mu \mathrm{m} \rightarrow 1 \\
\mathrm{~mm}\end{array}$ & Areia grossa & Areia grossa & Areia grossa \\
\hline $2 \rightarrow 1$ & $250 \rightarrow 500$ & Areia média & Areia média & Areia média \\
\hline $3 \rightarrow 2$ & $125 \rightarrow 250$ & Areia fina & Areia fina & Areia fina \\
\hline $4 \rightarrow 3$ & $63 \rightarrow 125$ & Areia muito fina & $\begin{array}{l}\text { Areia muito } \\
\text { fina }\end{array}$ & Areia muito fina \\
\hline $5 \rightarrow 4$ & $31 \rightarrow 63$ & Silte grosso & $\begin{array}{l}\text { Silte muito } \\
\text { grosso }\end{array}$ & $\begin{array}{c}\text { Silte muito } \\
\text { grosso }\end{array}$ \\
\hline $6 \rightarrow 5$ & $16 \rightarrow 31$ & Silte médio & Silte grosso & Silte grosso \\
\hline $7 \rightarrow 6$ & $8 \rightarrow 16$ & Silte fino & Silte médio & Silte médio \\
\hline $8 \rightarrow 7$ & $4 \rightarrow 8$ & Silte muito fino & Silte fino & Silte fino \\
\hline $9 \rightarrow 8$ & $2 \rightarrow 4$ & Argila grossa & Silte muito fino & Silte muito fino \\
\hline$>9$ & $<2$ & Ultra-argila & Argila & Argila \\
\hline
\end{tabular}

interpretação dos resultados. Por isso, a análise prévia da distribuição de freqüência das amostras é recomendável, através dos gráficos histograma e de outras técnicas gráficas disponíveis no SysGran.

A flexibilidade do SysGran pode ser explorada para comparações de métodos. É comum na literatura, por exemplo, comparações entre métodos gráficos e medidas dos momentos (Folk,
1966; Davis \& Ehrlich, 1970; Jaquet \& Vernet, 1976; Swan et al., 1978), mostrando as vantagens e desvantagens de cada um. Cada método dá uma ênfase diferente para diferentes partes da distribuição do tamanho do grão. $O$ método gráfico dá mais peso na porção central da curva de frequiência e menos nas caudas. $O$ limite inferior e superior e inferior dos percentís utilizados situase entre $16 \%$ e $84 \%$ para Folk \& Ward (1967) e 5\% e 95\% para 


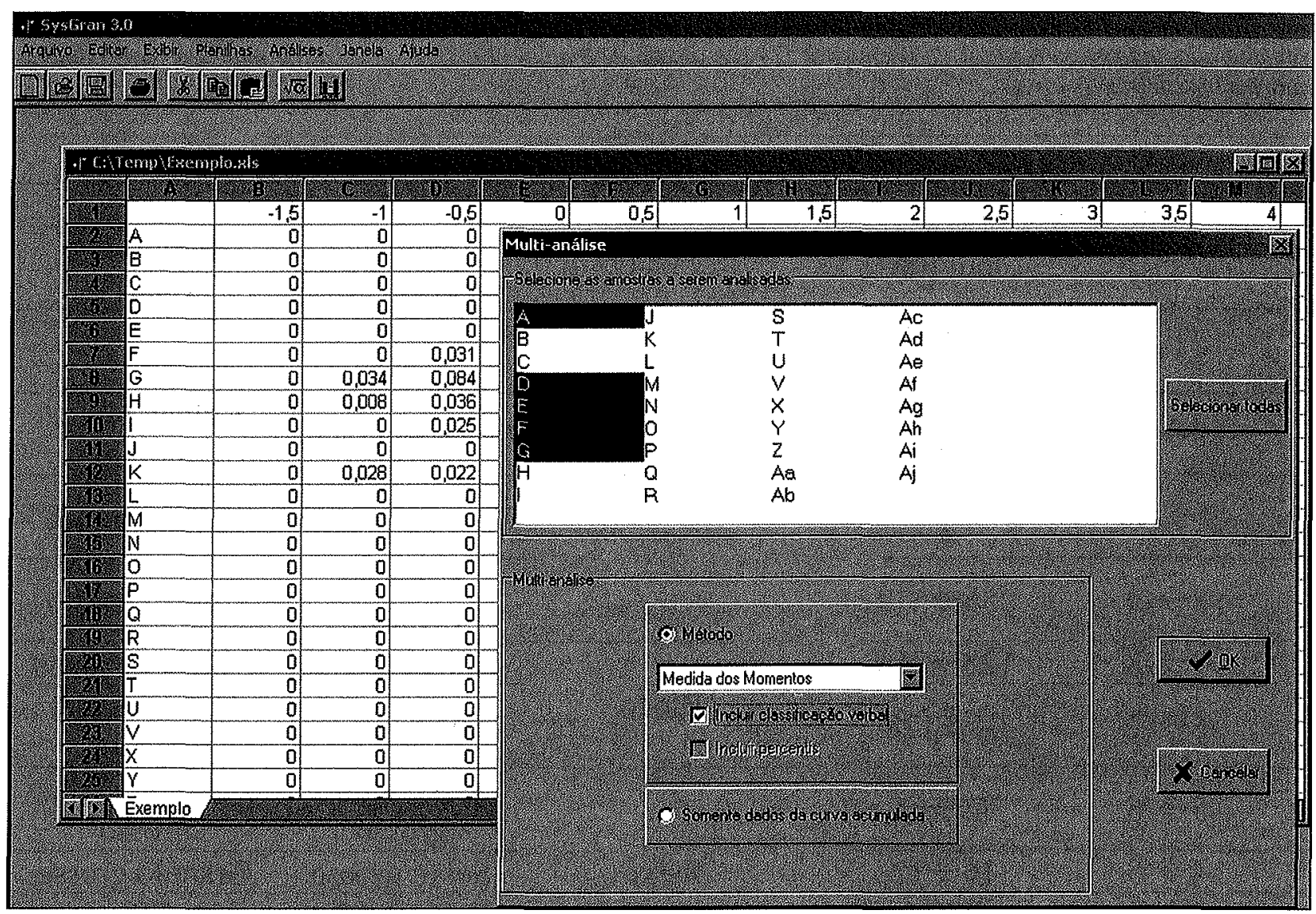

Figura 1. Exemplo de tela do SysGran: opções de análises estatisticas e dados brutos.

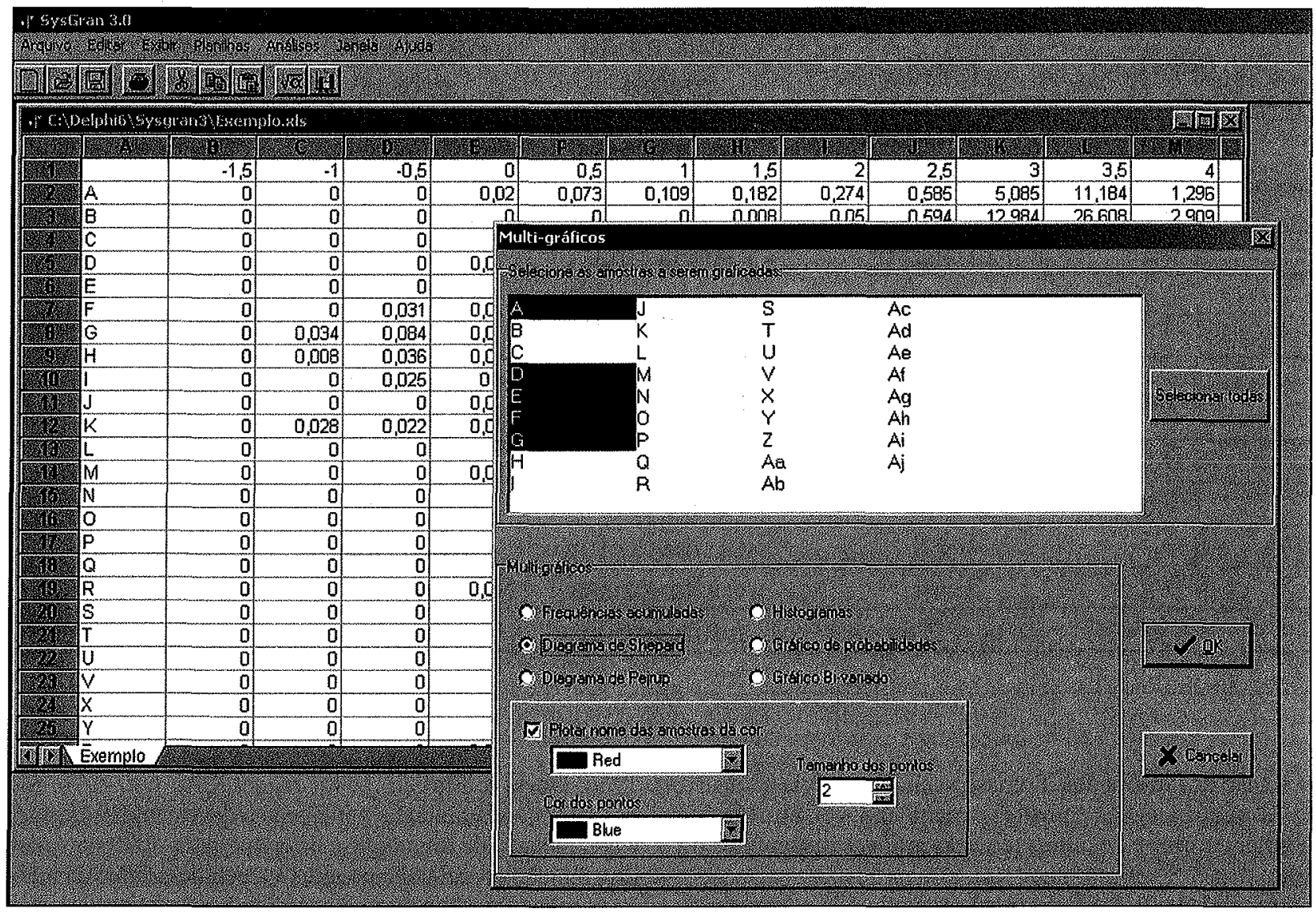

Figura 2. Exemplo de tela do SysGran: opções de gráficos. 


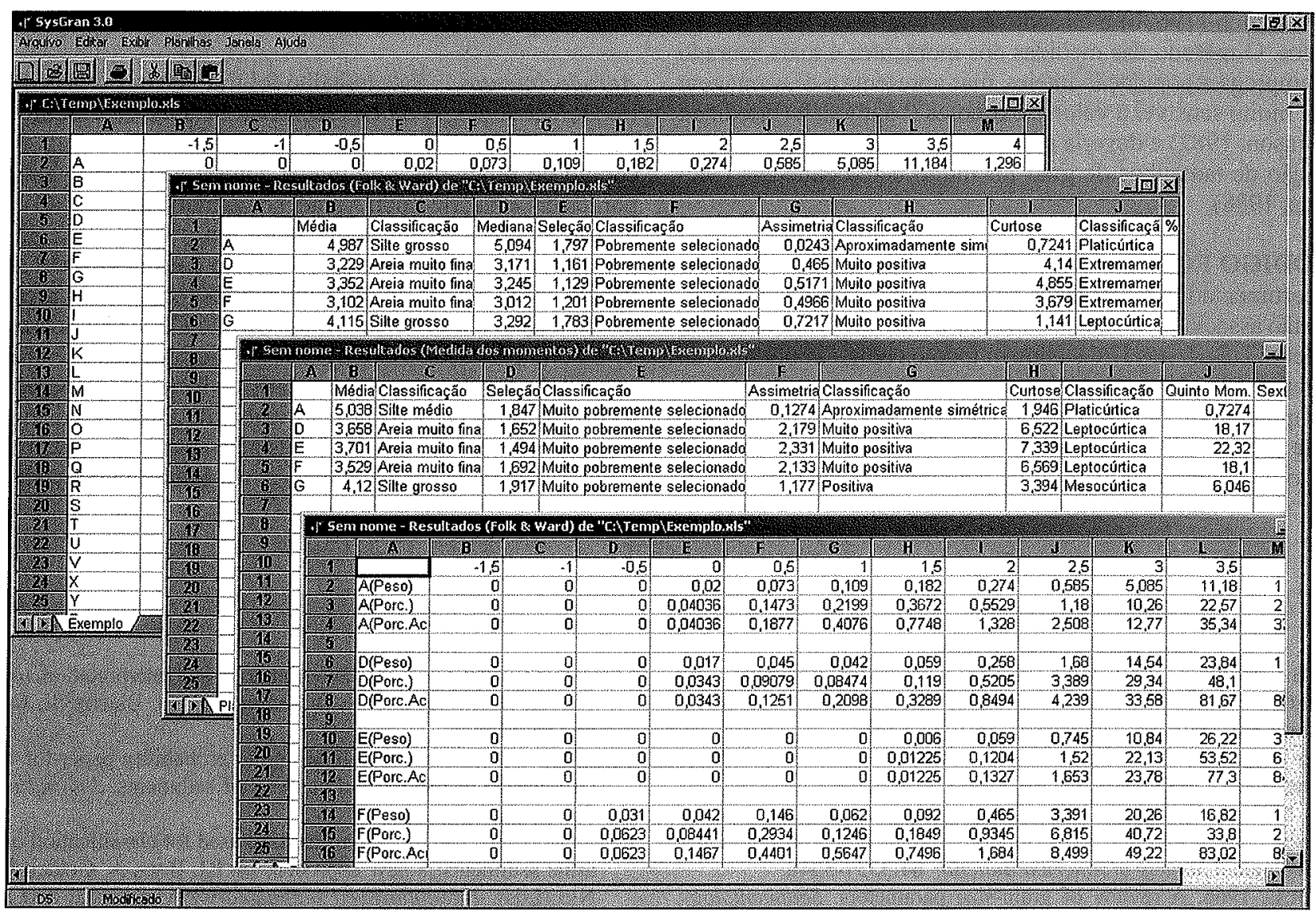

Figura 3. Exemplo de tela do SysGran: telas de planilhas de saída de resultados.

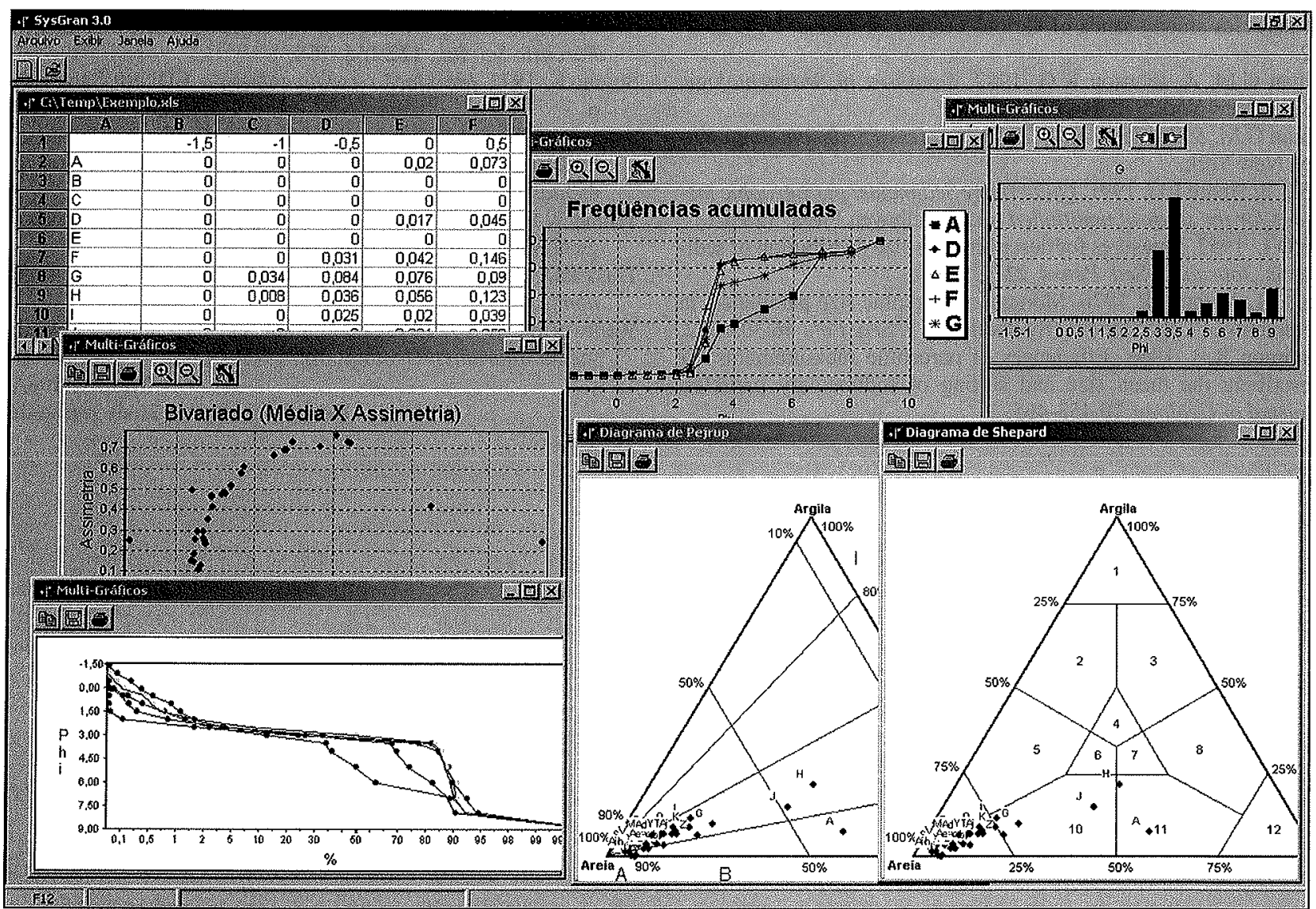

Figura 4. Exemplo de tela do SysGran: telas de saidas de gráficos 
MacCammon (1962b) e os sedimentos fora desta faixa são ignorados. $\mathrm{O}$ método das medidas dos momentos é mais acurado pois emprega a população inteira, mas apresenta a desvantagem de ser muito sensível a valores extremos ("outliers") (Friedman \& Johnson, 1982) e deveria ser aplicado somente quando a distribuição de tamanhos de grão for muito bem conhecida (McManus, 1988).

O CÓDIGO FONTE O código do programa é modulado e compartimentado em unidades de texto (Units) que quase sempre estão associadas a uma janela onde a interação com o usuário acontece. Três destas Units contêm todo o código importante do programa: PlaniUni.pas, Unit1.pas e GrafUni.pas. O programa é compilado em Delphi 5 tendo como pré-requisitos apenas o pacote gratuito de componentes RX Lib (atualmente parte do projeto de código aberto JEDI de componentes Delphi - www. sourceforge.jedi.org)

Agradecimentos Este trabalho foi fruto de uma bolsa de doutorado em zoologia na Universidade Federal do Paraná, realizado no Centro de Estudos do Mar em Pontal do Sul. Todo o código do programa é de minha autoria, tendo sido auxiliado por testers e na arte final. Agradeço do fundo do coração ao Anderson A. Pelanda pelo layout, pelos testes (beta, alfa e gama) e pelos inúmeros comentários; ao Kassio Rios pelo BMP da tela inicial, ícone do programa, configuração original do programa de instalação e testes (gama); ao vizinho Carlos R. Soares pela assessoria para sedimentologia, sugestões originais de análises, intermediação de contatos; ao Carlos Alberto Borzone pela detecção de bugs na classificação verbal do método das medidas dos momentos; à Paranaguá Pilots pelo auxílio financeiro ao laboratório de modelagem ecológica; e à Eunice pela paciência.

\section{Referências}

Blott S.J. \& Pye K. 2001. GRADISTAT: a grain size distribution and statistics package for the analysis of unconsolidated sediments. Earth Surf. Process. Landforms, 26:1237-1248.

Carver R.E. (edt.) 1971. Procedures in sedimentary petrology. WileyInterscience, $653 \mathrm{pp}$.

Davis M.W. \& Ehrlich R. 1970. Relationships between measures of sediment-size-frequency distributions and the nature of sediments. Geological Society of America Bulletin, 81:3537-3548.

Folk R.L. 1966. A review of grain-size parameters. Sedimentology, 6:73-93.

Folk R.L. \& Ward W.C. 1957. Brazos River bar: a study in the significance of grain size parameters. Journal of Sedimentary Petrology, 27:3-26.

Friedman G.M. 1979. Differences in size distributions of populations of particles among sands of various origins. Sedimentology, 26:332.

Friedman G.M. \& Johnson K.G. 1982. Exercises in Sedimentology. Wiley, New York.

Friedman G.M. \& Sanders J.E. 1978. Principles of Sedimentology. Wiley, New York.

Gale S.J. \& Hoare P.G. 1991. Quaternary Sediments: petrographic methods for the study of umlithified rocks. Belhaven Press. $323 \mathrm{p}$.

Inman D.L. 1952. Measures for describing the size distribution of sediments. Journal of Sed. Petrol. 22:125-145.

Jaquet J.M., Vernet J.P. 1976. Moment and graphic size parameters in sediments of Lake Geneva (Switzerland). J. Sed. Pet., 46:305-312.

McCammon R.B. 1962. Efficiences of percentile measurements for describing the mean size and sorting of sedimentary particles. Journal of Geology, 70:453-465.
McManus J. 1988. Grain size determination and interpretation. in: M.E Tucker. (Ed.): Techniques in Sedimentology. Blackwell, Oxford, pp.: 63-85.

Otto G.H. 1939. A modified logarithmic probability paper for the interpretation of mechanical analysis of sediments. Journal of Sed Petrol, 9:62-76.

Pejrup M. 1988. The triangular diagram used for classification of estuarine sediments: a new approach. In: P.L Boer, A. Gelder \& S.D. Nio (Eds). Tide-influenced sedimentary environments and facies. D. Reidel Publishing Company. Holland.

Pye K. \& Blott S.J. 2004. Particle size analysis of sediments, soils and related particulate materials for forensic purposes using laser granulometry. Forensic Sci. Int., 144:19-27.

Sugita R. \& Marumo Y. 2001. Screening of soil evidence by a combination of simple techniques: validity of particle size distribution. Forensic Sci. Int., 122:155-158.

Suguio K. 1973. Introdução à sedimentologia. EDUSP, São Paulo, 317 p.

Swan D., Clague J.J., Luternauer J.L. 1978. Grain size statistics I: evaluation of the Folk and Ward graphic measures. Journal of Sedimentary Petrology, 48:863-878.

Tanner W.F. 1995. Environmental clastic granulometry. Florida Geological Survey, Special Publication No 40.142 p.

Trask P.D. 1930. Mechanical analysis of sediments by centrifuge. Economic geology, 25:581-599.

Wentworth C.K. 1922. A scale of grade and class terms for clastic sediments. Journal of Geology, 30:377-392

Manuscrito A-1640

Revisão aceita em 26 de junho de 2006 